\title{
Methodologies and proposals to facilitate the integration of small and medium consumers in smart grids
}

\author{
Carlos Álvarez ${ }^{\boxplus}$, José I. Moreno ${ }^{2}$, Gregorio López², Camilo Carrillo3, \\ Ignacio J. Ramírez ${ }^{4}$, Javier Matanza ${ }^{5}$, Sergio Valero-Verdu ${ }^{6}$, \\ Antonio Gabaldón 7 , MariCarmen Ruiz ${ }^{8}$ \\ ${ }^{1}$ Institute for Energy Engineering, IIE-UPV, Valencia, Spain \\ ${ }^{2}$ Telematics Engineering Department, Universidad Carlos III de Madrid, Spain \\ ${ }^{3}$ Dpt. Of Electrical Engineering, Universidad de Vigo, Vigo, Spain \\ ${ }^{4}$ Dpt. Of Electrical Engineering, Universidad de Zaragoza, Zaragoza, Spain \\ ${ }^{5}$ Institute for Research in Technology, ICAI School of Engineering, IIT-ICAI, Madrid, Spain \\ ${ }^{6}$ Dpt. Of Mechanical Engineering and Energy, Universidad Miguel Herńndez de Elche, Elche, Spain \\ ${ }^{7}$ Dpt. Of Electrical Engineering, ETSII, Universidad Politécnica de Cartagena, Cartagena, Spain \\ ${ }^{8}$ Dpt. Of Applied Mathematics and Statistics, Universidad Politécnica de Cartagena, Cartagena, Spain \\ $凶$ E-mail: calvarez@die.upv.es
}

\begin{abstract}
Future power grids need to be flexible on the demand side to develop a credible energy policy, and in particular the integration of renewable. This objective will need a more active consumer. The demonstration of customer capabilities is an important challenge for small and medium-sized segments, since their potential (contribution to load curve) is undoubtedly of interest. REDYD-2050 (http://www.redyd2050-der.eu/) is a research network funded by Spanish Government (2015-2017) that integrates seven groups that develop research in key technologies to achieve an integral development of demand response (DR). This article presents the objectives of the network, including integrating technologies and proposing innovative solutions to DR concerns such as modelling and aggregation, automation, application of ICT, implementation in markets, price and consumption forecasts, or monitoring and verification.
\end{abstract}

\section{Introduction}

The purpose of REDYD-2050 is to exchange knowledge and experiences that will support the development and deployment of demand response (DR) in power systems, while demonstrating the DR's technical capacity face to the more 'conventional' supply-side resources. The principle of equality in capabilities must be demonstrated and verified for DR (for example, it has been established in US regulations [1, 2]), and this issue needs the development of new methodologies and proposals. The so-called 'Energy Union' is one of the thematic priorities for the European Commission (EC), where DR could play a key role in energy policy. EC states (see the Energy Market Framework Strategy Report to European Parliament, [3]) that the future electricity market should have at its core an active consumer taking advantage of new technology to reduce their costs, and allowing that customer fully participate in the energy transition. From the economic point of view, the energy commissioner stated [4] that 'Kthe business case for more active participation of demand is clear - demand side response alone could save our economy up to 100 Billion Euro per yearK'. Small- and medium-users account for more than $50 \%$ of consumption, so their contribution to this savings is of high economic interest, and they need a demonstration and in-depth exploration of their possibilities. The situation in other countries is similar. In the USA the response is low but the potential, if developed, can be high (i.e. around $60 \%$ of peak reduction can be achievable from the participation of small segments [5]). To help answer what remains to be done with DR, working groups were formed by the Department of Energy and The Federal Energy Regulatory Commission in four areas [5]: cost effectiveness, measurement and verification, programme design and implementation and tools and methods. The rest of this paper is devoted to show REDYD-2050 contributions and future development in some of these areas.

\section{Research areas of interest}

Exploiting DR resources in small customer segments has three major advantages: different kinds of capability and availability; geographical distribution of the resource and reliability (a large amount of resources with similar potentials). However, it has serious drawbacks: how to select resources (identification of demand patterns, monitoring and segmentation of demand), how to validate the flexibility and the response of those resources (modeling and control of loads), the conversion of the consumer into 'prosumer' (prediction of demand and generation, and their management), how to inform and, at the same time, guarantee the security of that information (education, cyber-security), how to add demand enabling the performance of aggregators (and curtailment service provider (CSP), balance responsible parties (BRP) or load serving entity (LSE)) and, finally, the improvement of DR cost-effectiveness (response optimisation, information communication technology (ICT) management, price prediction, eMKT market synergies with energy efficiency). Due to the aforesaid arguments, DR 'problem' is so complex that it is impossible at the department or institute levels achieve an optimal resolution. This makes necessary the collaboration of a network of experts in several of these fields, in a way that allows the ensemble to be competitive at international level. Fig. 1 shows the 'specialties' and synergies expected to be jointly developed by the seven groups that integrate REDYD-2050. 


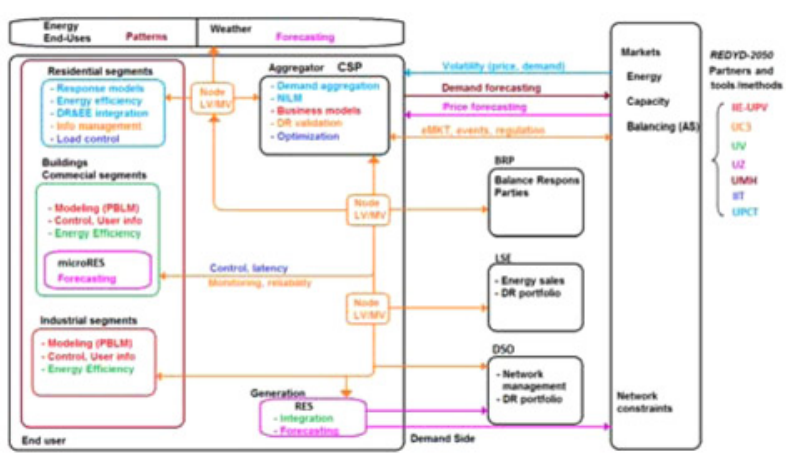

Fig. 1 Interrelation and interaction amongst different actors in DR according to REDYD's points of view

\section{The participation of active customers in electricity markets}

\subsection{Pros and cons of residential DR}

The situation of markets shows that small customers need some additional feedback: awareness of their contribution to system sustainability, understandable information from smart meters (SMs) or increased revenue from markets. With respect to this last remark, the information from markets (Fig. 2) points out that present revenue is mainly due to capacity options, but the role of elasticity of demand ought to be enhanced in future scenarios [6].

The possibility of increasing customer revenue arises as a way to reduce the incertitude with respect to new investments due to DR and, in this way, enhance energy and flexibility of demand. This could be achieved with the participation of customers in complex DR products (i.e. policies for both energy and ancillary services (AS) markets). Moreover, DR policies for these markets can share ICT and modelling methodologies. As in other markets, the participation of the customer needs a minimum level of demand flexibility (usually, some hundred of $\mathrm{kW}$ ). This is an important barrier for customer engagement, i.e. this participation needs to perform an aggregation of elemental demands. Moreover, the customer/aggregator requires an easy methodology to build and evaluate energy and cost curves, and estimate achievable revenue through DR and energy efficiency (EE).

\subsection{Modelling and aggregation}

The diversity in the approaches for these research areas deals both with the elemental load model and with the aggregation processes. With respect to elemental models, REDYD option is physically based load models, which involves the development of resistorcapacitor networks which characterise sub-models, representing energy flows between an appliance, the dwelling where the load renders the service and the environment. The overall model has several components (sub-models):

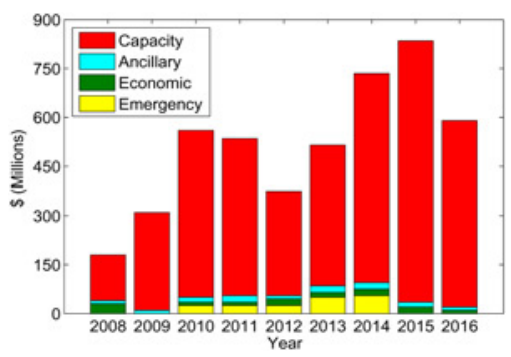

Fig. 2 PJM revenue for economic and load management DR markets. Adapted from [6] (2016 as of 11/2016)
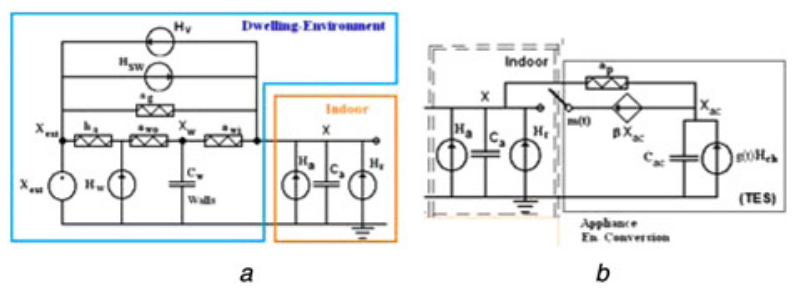

Fig. 3 Thermal-electrical analogy in Physically Load Based Methodology modelling: Elemental Dwelling and Appliance Submodels

(a) Dwelling sub-models and its environment, (b) Appliance energy conversion for TES loads

- Dwelling/environment sub-models (Fig. 3a), i.e. models that represent heat losses, through walls $\left(h_{a}, a_{w}\right)$, the floor $\left(a_{\mathrm{rg}}\right)$, windows $\left(a_{g}\right)$; as well as heat gains, solar radiation $\left(H_{\mathrm{sw}}\right)$, internal gains due to inhabitants/residents $\left(H_{r}\right)$ or appliances $\left(H_{a}\right)$ running inside the dwelling (i.e. lighting, TV). The model takes into account heat storage from the specific heat of walls $\left(C_{w}\right)$ and air mass $\left(C_{a}\right)$ (or roof/ground $\left(C_{\mathrm{rg}}\right)$ if necessary).

- The state variables that usually are temperatures: internal/indoor $(X)$, walls $\left(X_{w}\right)$ and roof/ground $\left(X_{\mathrm{rg}}\right)$.

- System inputs: external temperature $\left(X_{\text {ext }}\right)$ and heat sources: load conversion $\left(H_{\mathrm{ch}}\right)$, heat gains by radiation $\left(H_{\mathrm{sw}}, H_{\mathrm{w}}\right)$ or the work of other loads $\left(H_{a}\right)$.

Another model is the energy conversion into the appliance, i.e. electric power is converted to heat (space heating), 'cold' (air conditioning), or hot water (WH). This is represented in Fig. $3 b$ by a current source $\left(H_{\mathrm{ch}}\right)$.

Finally, there are one or several control mechanisms which decide if the load demands more or less power, i.e. a thermostat in some loads (these are $m(t)$ and $g(t)$ in Fig. $3 b$, driven by $X(t)$, the indoor temperature and $X_{\mathrm{ac}}(t)$ the temperature of storage materials such as bricks, ice, phase change materials,...).

With respect to the aggregation processes, they involve selecting loads with similar characteristics to achieve a minimum level of response $(\geq 100 \mathrm{~kW})$. Right now, the first alternative for the evaluation of aggregation is to write and solve equations for each load modeled in Figs. 3 and 4. Then, an average power for the aggregated load (through Monte Carlo simulation) is computed. This procedure needs the simulation of large numbers of loads (200-1000) and a lot of computation time (around 1-2 h). This procedure is feasible for DR in Energy Markets but not for AS markets, where the time for load response is in the range of minutes. Here, REDYD's proposal is to reduce the complexity of
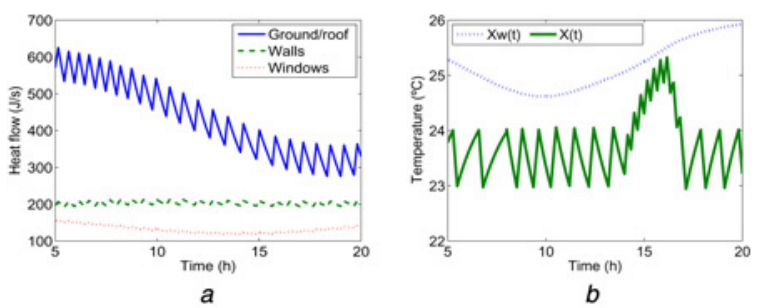

b
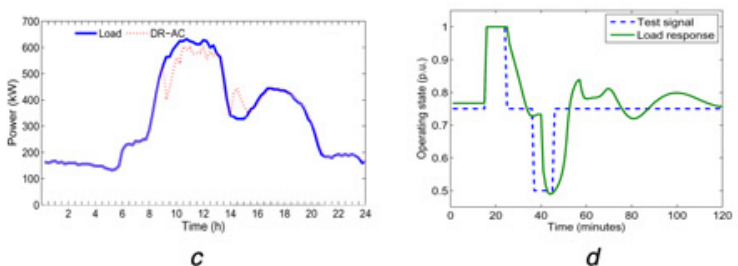

$c$

Fig. 4 Simulations of Aggregated and Elemental Models

(a) Heat flows resulting from the elemental model, (b) State variables (temperatures), (c) Aggregated response (Monte-Carlo) for DR price-response, (d) Aggregated response (Fokker-Planck) for AS 
the models (while balancing precision requirements) is using representative loads (average) whose probability density function is associated to stochastic partial differential equations. This can be obtained by Fokker-Planck (see [7]) in a shorter time (10-20 s or less). Fig. 4 shows an example of the possibilities of these models which are detailed in a international conference \& exhibition on electricity distribution (CIRED) companion paper.

\subsection{Feedback from SM}

The verification of load response is another important concern. At present, the capabilities of SM are underused. The customer should receive more information from SM to manage and take decisions with respect to DR and EE. Likewise, the aggregator should use this information to verify and certify the response. The proposal of REDYD is to use non-intrusive load monitoring (NIALM) methodologies to reduce the implementation costs of DR while taking profit from capabilities of existing resources (SM). NIALM approaches can be classified in micro and macro-scale [8]. Macro-scale option is compatible with the information supplied by SM without additional devices. HiddenMarkov chains are very popular methods in the literature [8] because they have a good performance and allow extracting patterns of elemental loads. Perhaps their main problem arises when loads are changing in patterns due to DR. To overcome this obstacle, integral transforms arise as an alternative to disaggregate typical DR loads (high-voltage alternative current and WH) [8]. These patterns can be used both for verifying load response and to fit parameters of the elemental models described in Section 4.

Another issue is customer segmentation. REDYD's team has experience in this field through the use of self-organised maps (SOM) [9]. The idea is to extract as much information as possible while compressing, filtering and simplifying the available information (daily, weekly demand) from SM. Perhaps, simpler input arrays to SOM which include relevant information about customer demand behaviour with additional information from transforms (e.g. Fourier for seasonal harmonics of load, and Hilbert information extracted from NILM process, peaks, load trailing, and leading edges etc.) would improve the segmentation process. This way, input vector supplied to SOM should include: the daily load demand, harmonic terms from weekly and monthly demand, and information about main loads extracted by NILM and their pattern of demand (discontinuities, end-use saturation etc.), with the aim of 'forcing' SOM networks to account not only for load behaviour (SM records) but also for load composition (when possible and in some extend according to NILM results).

\subsection{Economic models for $D R$ and EE}

It is necessary, in order to fully describe the offers resulting from DR and EE the evaluation of the involved costs and revenues, before the price for these offers can be set up. The value of each $\mathrm{kW}$ package ( $€ / \mathrm{kW}$-year) to be offered (in this example, for capacity markets), can be obtained by the (see coefficients in Table 1):

$$
\begin{aligned}
\text { Offer }= & {\left[\left(\mathrm{CA}+\mathrm{IC} * \mathrm{cic}+\sum_{k}\left(\mathrm{ICT}_{k} * \mathrm{citc}_{k}\right)\right.\right.} \\
& -\mathrm{INC}+\mathrm{AGG} * \text { life })-\left(\sum_{i=1}^{\text {life }}\left(\mathrm{ENER}^{\mathrm{i}} * \text { price }_{i}\right)\right. \\
& +\mathrm{AIC}+\mathrm{OM} * \text { life })] \frac{1}{\text { PWR } * \text { cmyears }}
\end{aligned}
$$

Fig. 5 shows the evaluation of these offers according to some of the parameters considered in

(1). Power is computed with the previously described models.

As shown in Fig. 5, the value of these offers and their effectiveness depends during its lifetime on the behaviour of
Table 1 Coefficients for the economic model (1)

\begin{tabular}{lc}
\hline Short name & Description \\
\hline CA & $\begin{array}{c}\text { initial (capital) costs } \\
\text { IC, AIC }\end{array}$ \\
ICT & Installing and avoiding installing costs \\
Cic & installing coefficient (\% attributable to DR) \\
OM & operating and maintenance \\
PWR, ENER & change in power or energy due to DR\&EE \\
INC & incentives, subsidies \\
AGG & aggregator costs \\
life & lifespan of DR or EE action \\
price & energy price \\
cmyears & operational lifetime of policy in market \\
\hline
\end{tabular}
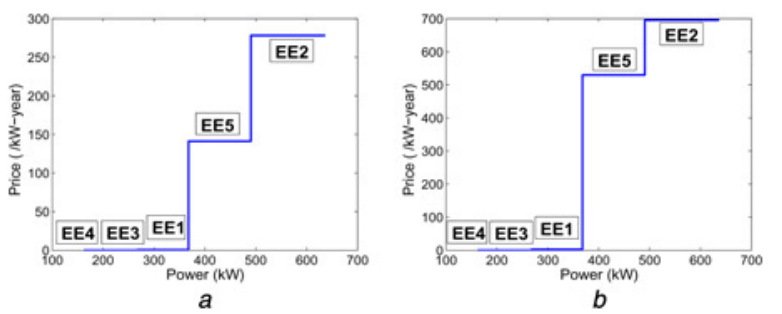

Fig. 5 Offers' elasticity due to 'cmyears' parameter,

(a) Revenues during all the lifetime of EE policy, (b) Revenues during 5 years

markets price. In this way, and to avoid further problems, it is necessary to consider problems such as price forecasting and market's volatility. The team of REDYD-2050 has developed some tools to perform energy price forecasting based on non-parametric estimation and neural networks [10]. The study of volatility and market dependencies has been performed through the so-called permutation entropy [11]. This method allows dealing with the seasonal component of the price series before the analysis, improving the capability of the method for the detection of changes in dependence levels along time through the filtering of seasonal patterns in prices (for instance, daily and weekly).

\section{Challenges and trends in ICT for residential DR}

ICT plays a key role when implementing DR programmes for small and medium customers in practice.

From the communications perspective, as Fig. 6 illustrates, on the one side, there are different types of DR with different temporal requirements, ranging from seconds or a few minutes to even days. On the other side, there are many communications technologies available in the market that presents different features in terms of quality of service, ranging from low-cost low-power low-data-rate technologies to very sophisticated and expensive communications infrastructures providing high data rate ultra-connectivity. Such communications technologies can be combined or not leading to
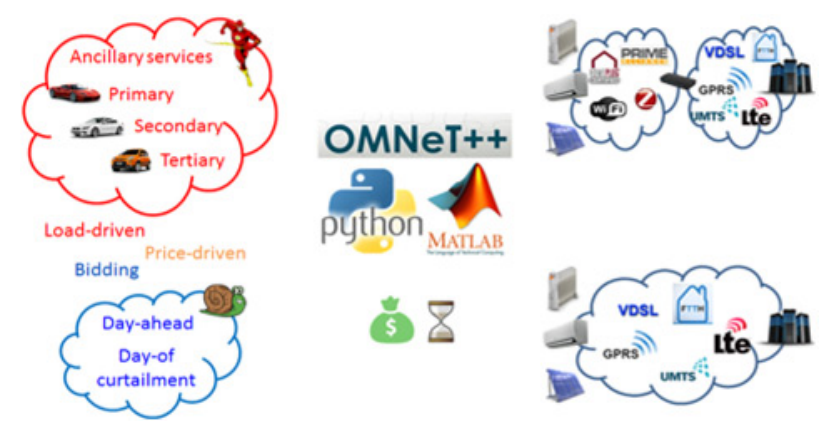

Fig. 6 Communication network simulation tools and frameworks represent a cost-effective solution to make decisions on the suitability of communications architectures and technologies to DR programmes 
hierarchical or monolithic communications architectures, each of them presenting pros and cons. Typically monolithic approaches facilitate the management of the communications infrastructure and favour security, but they lack flexibility, increase operational costs and may present technical problems [12]; whereas managing heterogeneous communications infrastructures is more complex and they present more potential points of attack, but they boost flexibility and scalability and make a more efficient use of communications media by aggregating data [13]. Therefore, communications network simulation tools and frameworks are required to allow selecting the most appropriate communications architecture and technologies for a specific situation [14], as well as evaluating the performance of a given communications infrastructure under certain conditions [15], without incurring in the high costs of making such decisions based on pilot schemes and in-field tests. Hence, this represents a remarkable research line within REDYD2050 project, which is indeed on-going [16].

However, DR programmes not only depend on the state of the power grid and on how the communications network works, but also on many other factors, such as building thermal response or meteorology. As a result, in order to provide a more holistic approach, different simulation tools can be combined, using standard architectures or interfaces such as high-level architecture or functional mockup interface, leading to the so-called co-simulation tools and frameworks [17], such as the one shown in Fig. 7. Thus, this represents yet another research line where REDYD2050 team already gathers experience [18].

As it has already been said, bringing DR to small- and medium-customers presents benefits from different perspectives, but it also presents technological challenges. Form the information technology (IT) perspective, in particular, bringing monitoring and control networks down to the SMs and beyond increases the number of devices and the amount of data that need to be processed dramatically, putting traditional information systems on the ropes. Therefore, emerging cutting-edge IT technologies such as big data and cloud computing are key in order to achieve handling such a huge amount of data, from different types and in different formats, in the very constrained temporal conditions that some DR programmes entail $[19,20]$. As a result, exploring the application of these technologies to the specific case of DR also falls within the research interests of the REDYD2050 project.

Although, as it has just been shown, ICT present a great potential and provide key features to make the DR dream come true, but with great power comes great responsibility, and they also bring great challenges and risks, cybersecurity standing out among them all. As a matter of fact, such a critical infrastructure as the electrical grid will have ever been as exposed as when fully digitalised. As a result, cybersecurity in smart grid has been a hot topic for a long while $[21,22]$ and it is even more critical if DR is in play (e.g. imagine the disastrous effects in power delivery that could cause an attacker who takes control of a hypothetical DR platform to match consumption and generation at the level of small and medium consumers). Therefore, the REDYD2050 project also aims to investigate potential risks and attacks to this kind of

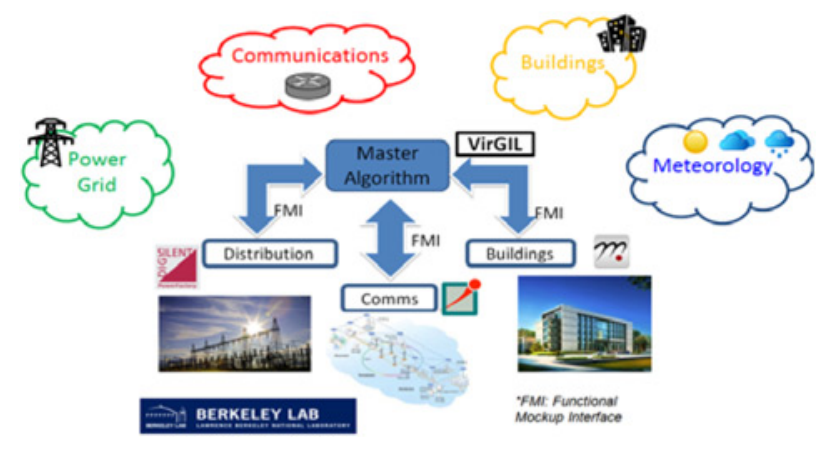

Fig. 7 Co-simulation architecture of the VirGIL tool developed by the Lawrence Berkeley National Laboratory [18] infrastructures, as well as possible solutions and best practices that mitigate such risks.

\section{Conclusions}

Consumer participation in electricity networks is of growing interest in the EU. The complexity of the problem makes it necessary to share and discuss experiences amongst researchers from different academic disciplines, consumers and, in general, agents and operators of networks and markets. The sharing of experiences within the framework of REDYD-2050 platform, and its dissemination, can contribute to improvements in the operation and planning of DR into our systems. This paper presents different methodologies and tools to develop a model for the effective engagement of customers and aggregators (and other agents) in DR.

\section{Acknowledgment}

This work was supported by the Spanish Government (Ministerio de Economía, Industria y Competitividad) by grant no. ENE2015-70032-REDT.

\section{References}

1 FERC. Orders 745\&755: 'DR compensation in organized wholesale energy markets', 2011

2 FERC. Orders 745\&755: 'Frequency regulation compensation in organized wholesale power markets', 2011

3 COM 339 final: 'Communication from the EC on a new deal for energy consumers', 2015

4 Arias, M.: 'A modern energy union for competitive, secure and sustainable energy for the European Industry'. Proc. of 2015 Euro Economic Congress, Poland, 2015

5 DoE, FERC: 'A national forum on demand response'. Available at https://www ferc.gov/industries/electric/indus-act/demand-response/dr-potential.asp

6 PJM Demand Side Response Operations: 'Markets activity report: Nov 2016'. Available at https: \www.pjm.com

7 Alvarez, C., Gabaldón, A., Molina, A.: 'Assessment and simulation of the responsive demand potential in end-user facilities: application to a university customer', IEEE Trans. PWRS, 2004, 19, (2), pp. 1223-1232

8 Gabaldón, A., Molina, R., Marín-Parra, A., et al.: 'Residential end-uses disaggregation and demand response evaluation using integral transforms', J. Mod. Power Syst. Clean Energy, 2017, 5, (1), pp. 1-14

9 Valero, S., García, M.O., Senabre, C., et al:: 'Classification, filtering and identification of electrical customer load patterns through the use of self-organizing maps', IEEE Trans. Power Syst., 2006, 21, (4), pp. 1672-1682

10 Gabaldón, A., Guillamón, A., Ruiz, M.C., et al.: 'Development of a methodology for clustering electricity-price series to improve customer response initiatives', IET Gener. Trans. Distrib., 2010, 4, (6), pp. 706-715

11 Ruiz-Abellón, M.C., Guillamón, A., Gabaldón, A.: 'Dependency-aware clustering of time series and its application on energy markets', Energies, 2016, 9, (10), p. 809

12 Mao, R., Julka, V.: 'Wireless broadband architecture supporting advanced metering infrastructure'. Proc. of IEEE 73rd Vehicular Technology Conf. (VTC Spring), Budapest, Hungary, 2011

13 López, G., Moreno, J.I., Amaris, H., et al.: 'Paving the road toward smart grids through large-scale advanced metering infrastructures', Electr. Power Syst. Res., 2015, 120, pp. 194-205

14 López, G., Moura, P., Moreno, J.I., et al.: 'Multi-faceted assessment of a wireless communications infrastructure for the green neighborhoods of the smart grid', Energies, 2014, 7, pp. 3453-3483

15 Matanza, J., Kiliccote, S., Alexandres, S., et al.: 'Simulation of low-voltage narrow-band power line communications networks to propagate open ADR signals', J. Commun. Netw., 2015, 17, pp. 656-664

16 Seijo, M., López, G., Matanza, J., et al.: 'Planning and performance challenges in power line communications networks for smart grids', Int. J. Distrib. Sens. Netw., 2016, 2016, pp. $1-17$

17 Mets, K., Aparicio Ojea, J., Develder, C.: 'Combining power and communication network simulation for cost-effective smart grid analysis', IEEE Commun. Surveys Tutor., 2014, 16, pp. 1771-1796

18 Chatzivasileiadis, S., Bonvini., M., Matanza, J., et al.: 'Cyber physical modeling of distributed resources for distribution system operations', IEEE Proc., 2015, 104, pp. 789-806

19 Shyam, R., Bharathi Ganesh, H., Sachin Kumar, S., et al.: 'Apache spark a big data analytics platform for smart grid', Procedia Technol., 2015, 21, pp. 171-178

20 Bera, S., Misra, S., Rodrigues, J.J.: 'Cloud computing applications for smart grid: a survey', IEEE Trans. Parallel Distrib. Syst., 2015, 26, pp. 1477-1494

21 Metke, A. R., Ekl, R.L.: 'Security technology for smart grid networks', IEEE Trans. Smart Grids, 2010, 1, pp. 99-107

22 Liu, J., Xiao, J., Li, S., et al.: 'Ciber security and privacy issues in smart grids', IEEE Commun. Surveys Tutor., 2012, 14, pp. 981-997 